\title{
Yarkovsky Effect on the Orbital Dynamics of 1566 Icarus Asteroid
}

\author{
Niken Rara Galih Amithya Parastuti ${ }^{1 *}$, Endang Soegiartini ${ }^{1}$

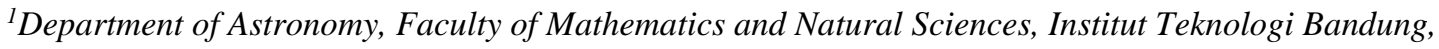 \\ Jl. Ganesa 10, Bandung - 40112, Indonesia
}

( Received: May 9, 2016, Revised: July 30, 2016, Accepted: January 16, 2019 )

\begin{abstract}
The orbital dynamic of small objects is an n-body problem that can not be solve by analitically, it is needed to use numerical integration to find the solution instead. This work is about orbital dynamic of asteroid 1566 Icarus under Classical Newtonian gravitation and if thermal effect (Yarkovsky effect) is included. Yarkovsky Effect is a thermal radiation force resulted from time span of small rotating objects to receive heat from the Sun and then re-radiates it. The Yarkovsky Effect is working optimum for objects with diameter from $10 \mathrm{~cm}$ up to $10 \mathrm{~km}$, and now is implemented to Asteroid 1566 Icarus with diameter $1.3 \mathrm{~km}$ which are member of Apollo and Earth crosser object. This Asteroid is called Earth crosser due to its orbit is crossing Earth's orbit. With semi major axis $a \approx 1.078$ au and eccentricity $e \approx 0.827$, asteroid 1566 Icarus has perihelion distance $q=0.18674$ au or less than semi major axis of Mercury. Due to that reason, Yarkovsky effect was considered to be applied on the orbital dynamics of asteroid 1566 Icarus. Due to sensitivity in input-data of numerical integration for n-body, one hundred simulation preliminary data were made as input in numerical integration process, therefore, 100 clones of Asteroid 1566 Icarus are gathered. Cloning process was conducted by using random number from Asteroid 1566 Icarus orbital elements at epoch 2456800.5 (23 May 2014) to standard deviation $\sigma$. The integration then later conducted within $10^{5}$ years time span from the epoch. The result shown that the orbital dynamics of asteroid 1566 Icarus with Yarkovsky effect is still within the range of 100 clones of asteroid 1566 Icarus. Thereby, within $10^{5}$ years, Yarkovsky effect does not change the orbital dynamic of asteroid 1566 Icarus globally, except for two phenomenon between close encounter with planet.
\end{abstract}

Keywords: Asteroid, 1566 Icarus, Yarkovsky effect, classical Newtonian

\section{INTRODUCTION}

All object in the Solar System moves around the Sun under influences of Newtonian-gravitational from the Sun and 8 planets. Apart from the gravitational effect, non-gravitational effect such as thermal radiation, also affects all the member of Solar System. Those effects influence the movement and the behaviour of orbital dynamics of small body objects in the Solar System. In this paper, we woud like to investigate the influence of Yarkovsky Effect, a non-gravitational effect in small body object in the Solar System [3]. In this paper, we propounds the difference of orbital dynamics of Asteroid 1566 Icarus which is only influenced by Newtonian

\footnotetext{
* Corresponding author

E-mail address: nixraragalih@gmail.com
}

gravitation and by including Yarkovsky effect, within $10^{5}$ years span.

\section{Orbital element of asteroid 1566 Icarus}

The position of a celestial object is represented by orbital element.

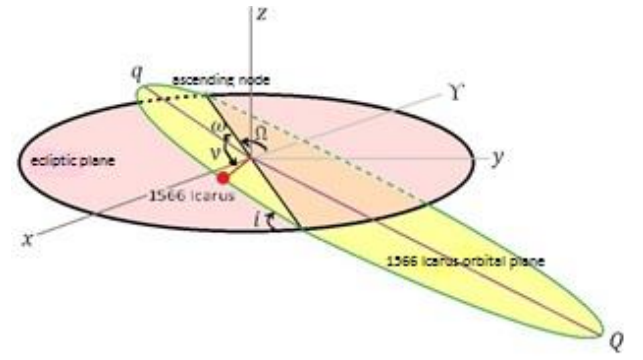

Figure 1. The scheme of orbital element of asteroid 1566 Icarus from epoch 2456800.5 (23 May 2014) 
Fig.1 shows the diagram of orbital element of asteroid 1566 Icarus at epoch 2456800.5 (23 May 2014). The orbital plane of asteroid 1566 Icarus is shown by the yellow-colored, and the Earth's orbit as plane of reference is shown by ecliptic rose-colored. The direction of the first point of Aries, as the references point, is written by $\Upsilon$. The semi major of this orbit is denoted by $a$, the inclination angle or the angle which is formed by ecliptic plane and orbital plane of celestial object, is denoted by $i$, and the eccentricity of the orbit is denoted by $e$. The perihelon and aphelion distance of the object are represented by $q$ and $Q$, which is the shortest and longest distance from the Sun. Meanwhile $\omega, \Omega$, and $v$ are argument of perihelion, longitude of ascending node, and true anomaly. Table 1 below shows us the orbital element of asteroid 1566 Icarus and 8 planets at epoch 2456800.5 (23 May 2014) [8].

Table 1. Orbital element of asteroid 1566 Icarus and 8 planets from epoch 2456800.5 (23 May 2014) [8].

\begin{tabular}{cccccccccc}
\hline No & Object & $a[\mathrm{au}]$ & $e$ & \multicolumn{1}{c}{$i\left[^{\circ}\right]$} & $\omega\left[^{\circ}\right]$ & $\Omega\left[^{\circ}\right]$ & $M\left[^{\circ}\right]$ & $q[\mathrm{au}]$ & $Q[\mathrm{au}]$ \\
\hline 1 & 1566 Icarus & 1.07802 & 0.82677 & 22.83010 & 31.3542 & 88.0275 & 54.9592 & 0.18674 & 1.96931 \\
2 & Mercury & 0.38710 & 0.020564 & 7.00411 & 29.1668 & 48.3131 & 82.0573 & 0.30749 & 0.46670 \\
3 & Venus & 0.72333 & 0.00675 & 3.39451 & 54.6898 & 76.6408 & 190.6450 & 0.71845 & 0.72821 \\
4 & Earth & 1.00028 & 0.01673 & 0.02974 & 255.6660 & 208.8840 & 135.7380 & 0.98354 & 1.01702 \\
5 & Mars & 1.52362 & 0.09352 & 1.84844 & 286.5900 & 49.5148 & 253.3990 & 1.38114 & 1.66612 \\
6 & Jupiter & 5.20214 & 0.04888 & 1.30378 & 273.7790 & 100.5150 & 96.7589 & 4.94784 & 5.45644 \\
7 & Saturn & 9.54229 & 0.05472 & 2.48616 & 339.3910 & 113.6520 & 132.7290 & 9.02016 & 10.06443 \\
8 & Uranus & 19.18436 & 0.04803 & 0.77307 & 95.7373 & 73.8431 & 205.4820 & 18.26281 & 20.10591 \\
9 & Neptune & 30.00091 & 0.00932 & 1.76455 & 288.9210 & 131.7100 & 275.8240 & 29.72133 & 30.28050 \\
\hline
\end{tabular}

\section{Asteroid 1566 Icarus}

Asteroid 1566 Icarus is an asteroid with diameter $\approx 1.3 \mathrm{~km}$ and absolute magnitude $H \approx 16.9$ [8] and obliquity $\gamma=130^{\circ}$ [12]. With S-type asteroid but contains pyroxene and olive, this asteroid is SU; Q-type. The Bond-albedo of asteroid 1566 Icarus $\approx$ 0.33 [2].

As the member of Near Earth Asteroid (NEA), this asteroids be the most potentially hazardous or closely approach to the Earth. Based on its orbital element, NEA is divided into four groups, which are Amor, Apollo, Aten, and Atira [7] and written in Table 2. With $a \approx 1.078 \mathrm{AU}$ and $e \approx 0.827$, asteroid 1566 Icarus is Earth crosser object, which its orbit intersects with Earth's orbit from outside.

Table 2. NEA group

\begin{tabular}{cl}
\hline Group & \multicolumn{1}{c}{ Definition } \\
\hline Amor & $a>1.0 \mathrm{au}, 1.017 \mathrm{au}<q<1.3 \mathrm{au}$ \\
Apollo & $a>1.0 \mathrm{au}, q<1.017 \mathrm{au}$ \\
Aten & $a<1.0 \mathrm{au}, Q>0.983 \mathrm{au}$ \\
Atira & $a<1.0 \mathrm{au}, Q<0.983 \mathrm{au}$ \\
\hline
\end{tabular}

Fig. 2 show us the scheme of orbital diagram of NEA-group, and Fig. 3 show us the scheme of one orbital periode of asteroid 1566 (purple) and 4 other planets, i.e Mercury, Venus, the Earth, dan Mars (black).

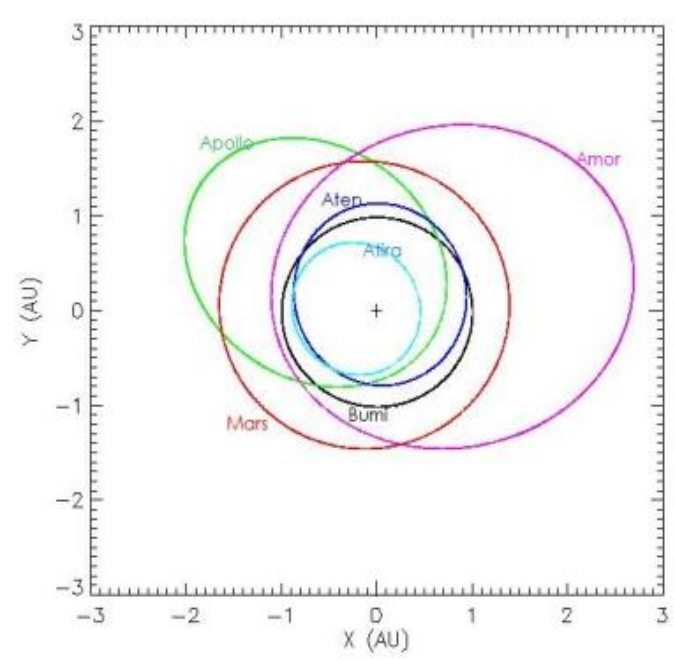

Figure 2. Orbital diagram of NEA-group [14]. 

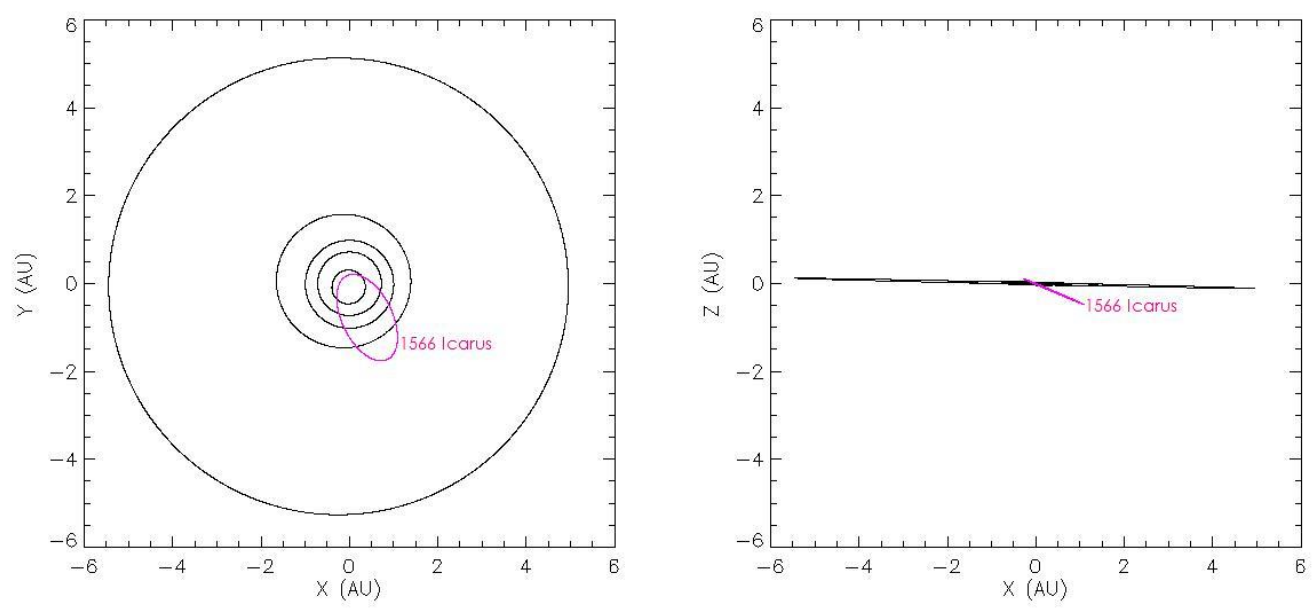

Figure 3. The scheme of one orbital periode of asteroid 1566 (purple) with Mercury, Venus, the Earth, dan Mars (inside to outside from the Sun, in point of 0,0$)$, in X-Y plane or ecliptic plane (left-side) and in X-Z plane or perpendicular to the ecliptic (right-side) [14].

\section{Yarkovsky Effect}

Yarkovsky effect was discovered by a Russian civil engineer, Ivan Osipovich Yarkovsky in 1888 [1]. Yarkovsky effect is a radiation force that is caused by the thermal effects in a long period of time which can change the orbital elements of a rotating body, in this case the small bodies, especially meteoroids and small asteroids in diameter of $10 \mathrm{~cm}$ to $10 \mathrm{~km}$ [3]. Fig. 4 shows us the scheme of Yarkovsky effect mechanism.

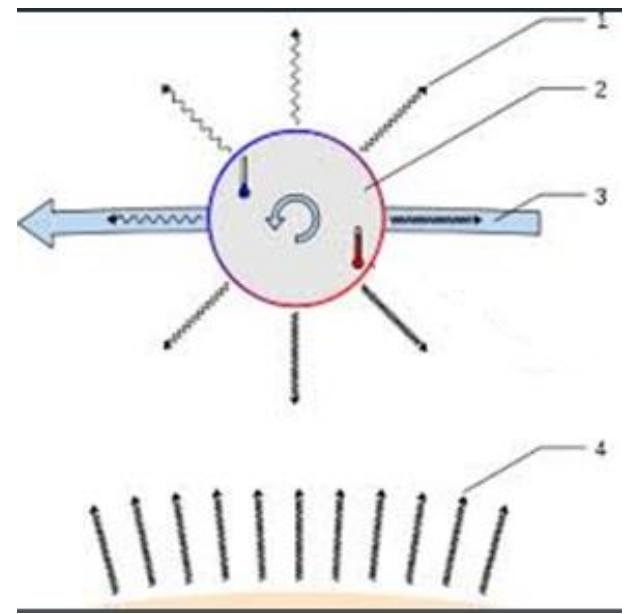

Figure 4. The scheme of Yarkovsky Effect Mechanism: 1. Radiant heat direction received by smaller object; 2 . The small body object; 3 . Orbit direction of small object; 4 . Sun radiant, red-color shows areas of small object with higher temperature, blue-color shows areas with lower temperature, arrow shows direction of smaller object's rotation (modified from [9])

In addition to relying on the size of the object, Yarkovsky effect also depends on the tilt of its rotation axis through normal vector of its orbit, often called obliquity $(\gamma)$. There are two components in Yarkovsky effect, which are daily component (diurnal) and seasonal. Diurnal component works optimum on object with the value of $\gamma=0^{\circ}$, with perpendicular rotation to the orbit. Since the effect correlates with the rotation of the object (one rotation equals to one day), this component is called daily component or diurnal component. Diurnal component causes the changes of $a$, increasing for objects with $\gamma<90^{\circ}$, or decreasing for $\gamma>90^{\circ}$ [3]. The seasonal component will be maximum at $\gamma=$ $90^{\circ}$, and causes the asteroid to move in spiral orbit, inward approaching to The Sun. With obliquity $\approx$ $103^{\circ}$, the seasonal component more dominant than diurnal component. [12].
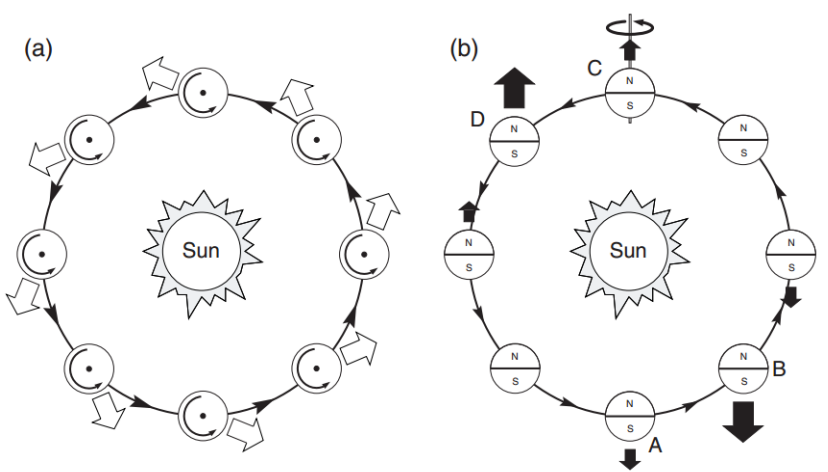

Figure 5. The scheme of Diurnal Yarkovsky effect and Seasonal Yarkovsky effect. (a) The diurnal Yarkovsky effect, with the asteroid's spin axis perpendicular to the orbital plane. The black arrows show the direction of rotation and revolution of small objects, while the white arrow indicates the magnitude and direction of the force that received of the object. (b) The scheme of seasonal component that works on small objects, with the asteroid's spin axis in the orbital plane (Bottke et al. 2002).

\section{Numerical Integration}

The numerical integration to investigate the orbital element dynamics for asteroid 1566 Icarus is executed using SWIFT integrator. SWIFT is a software designed by Hal Levison and Martin 
Duncan, specifically for numerical integration of solar system's body [10]. There are four integration techniques in SWIFT integrator, among others:

1. Wisdom-Holman Mapping (WHM), created by Jack Wisdom and Matt Holman

2. Regularized Mixed Variable Symplectic (RMVS), created by Hal Levison and Martin Duncan

3. $\mathrm{T}+\mathrm{U}$ Symplectic Orde 4 Method (TU4), created by Jeff Candy and W. Rozmus

4. Bulirsch-Stoer Method with the same input and output with WHM and three additional inputs. This method can also accommodate close intersect such as RMVS but ten times slower than RMVS.

We use RMVS method version 4 or RMVS4. This method was chosen due to availability close encounter for test particle, asteroid 1566 Icarus with major planets. RMVS4 method has been refined from its previous version which results in enabling integration more rapidly. However, RMVS4 method has not identified close encounter occurred within integration process, meanwhile Yarkovsky effect is using RMVS4 _ye. RMVS4 _ye integration method is the modified version of RMVS4 by additional instruction to accommodate Yarkovsky effect.

\section{NUMERICAL METHOD}

The Yarkovsky effect on the orbital dynamics of 1566 Icarus asteroid is investigated by added Yarkovsky effect equations into numerical integration of the Classical Newtonian equation. All the major planets from Mercury through Neptune were included as perturbing bodies, and the Earth is taken at the Earth-Moon barycenter as being one body, with the Moon's mass added to the Earth's.

\section{Mathematical Approach for Classical Newtonian}

Classical Newtonian represents n-body motion under gravitational force only. The equation for Classical Newtonian equation is written as follows:

$$
\begin{aligned}
m_{i} \ddot{\boldsymbol{r}}_{i} & =k^{2}\left[\sum_{i \neq j} \frac{m_{i} m_{j} r_{i j}}{r_{i j}{ }^{3}}\right] \\
\ddot{\boldsymbol{r}}_{i} & =\left[\sum_{j \neq i} \frac{k^{2} m_{j} r_{i j}}{r_{i j}{ }^{3}}\right]=\left[\sum_{j \neq i} \mu_{j} \frac{r_{i j}}{r_{i j}{ }^{3}}\right]=\left[\sum_{j \neq i} \mu_{j} \frac{\left(r_{j}-\boldsymbol{r}_{i}\right)}{r_{i j}{ }^{3}}\right]
\end{aligned}
$$

(2)

$$
\text { with }
$$$$
\mu_{i}=k^{2} m_{i} \text { dan } \boldsymbol{r}_{i j}=\boldsymbol{r}_{j}-\boldsymbol{r}_{i}
$$

In equation (1) and (2), $m_{i}$ and $m_{j}$ represents the mass of the- $i$ and the- $j$ object, $\ddot{\boldsymbol{r}}_{i}$ represents the acceleration of the-ith object and $r_{i j}$ represents the distance between $i$-th and $j$-th objects. On the other hand, $k$ is a Gaussian gravitational constant with $k=$ $\sqrt{G}=0.017202 a u^{3 / 2} M_{\odot} d a y^{-1}, G$ is gravitational constant.

\section{Mathematical Approach for Yarkovsky Effect}

Mathematically, Yarkovsky effect calculation is divided into two parts which are temperature distribution of object's surface and thermal radiation evaluation which resulted in force or torque [3]. To execute the surface temperature of the object, the heat diffusion equation is used for energy flows inside the object:

$\nabla \cdot(K \nabla T)=\rho C \frac{\partial T}{\partial t}$

or across its surface:

$\left(K \nabla T \cdot \boldsymbol{n}_{\perp}\right)+\varepsilon \sigma T^{4}=\alpha \varepsilon$

$K$ is thermal conductivity, $C$ is specific heat at constant pressure, $\varepsilon$ is surface termal emissivity, $\sigma$ is Stefan-Boltzmann constant, and $\alpha=1-A$ with $A$ is Bond-albedo. Equation (4) refers to surface element with normal vector $n_{\perp}$, and $\varepsilon$ is The Sun radiation flux through this element. Equation (1) and (2) can be solved numerically if $\varepsilon$ is known (which depends on the information of object's shape and rotation) and parameter values of $K, C$, and $\rho$ material.

To simplify the problem, we used some assumption for calculation. The non-linear form of surface thermal emission can be simplified with the assumption that temperature of the object is the average of the temperature, $T_{0}$, which can be written as:

$$
\begin{aligned}
& T=T_{0}+\Delta T \\
& \delta=\left(\Delta T / T_{0}\right) \ll 1
\end{aligned}
$$

If $T_{0}$ is constant, then the equation (3) and (4) can be written using $\delta$ variable, meanwhile in fourthorder within limit that can be written as :

$T^{4} \approx T_{0}^{4}(1+4 \delta+\cdots)$

To solve those equations, assuming isotropic emission, the force per unit mass is given by

$f=-\frac{2}{3} \frac{\varepsilon \sigma}{m c} \int_{S} d S(u, v) T^{4} n_{\perp}$

With $d S$ is the surface element within coordinate $(u$, $v$ ) or longitude and latitude in spherical system, $m$ is mass of the object and $c$ is velocity of light. Equation (8) can be solved by numerical integration or by fourth-order linearization assumption for temperature to execute analitical integration.

Yarkovsky acceleration can influence to small changes of $a$. Due to this small changes, the variations in $a$ can be write by $\frac{d a}{d t}$. If there is a spherical object with radius $R$ and ignoring exccentricity $e$, the average of daily and seasonal Yarkovsky effect interrupt is as follows: 


$$
\begin{aligned}
& \left(\frac{d a}{d t}\right)_{\text {diurnal }}=-\frac{8 \alpha}{9} \frac{\Phi}{n} F_{\omega}\left(R^{\prime}, \Theta\right) \cos \gamma+O(e)(9) \\
& \left(\frac{d a}{d t}\right)_{\text {seasonal }}=-\frac{4 \alpha}{9} \frac{\Phi}{n} F_{n}\left(R^{\prime}, \Theta\right) \sin ^{2} \gamma+O(e)
\end{aligned}
$$

The total $\frac{d a}{d t}$ is the super position of both $\frac{d a}{d t}$ values above, with $\Phi=\frac{\pi R^{2} \varepsilon_{0}}{m c}$ is the coefficient radiation pressure and $\gamma$ is obliquity.

The thermal parameter is represented by

$$
F_{v}\left(R^{\prime}, \Theta\right)=-\frac{\kappa_{1}\left(R^{\prime}\right) \Theta_{v}}{1+2 \kappa_{2}\left(R^{\prime}\right) \Theta_{v}+\kappa_{3}\left(R^{\prime}\right) \Theta_{v}^{2}}
$$

With $R^{\prime}$ is thermal wave $\left(R^{\prime}=R / l_{v}\right), R$ is radius and $l_{v}=\sqrt{\frac{K}{\rho C_{p} v}}$ is penetration depth. $\kappa_{1}, \kappa_{2}$ and $\kappa_{3}$ are analytic function of $R^{\prime}$. The thermal parameter is represented by $\Theta_{v}=\sqrt{K \rho C_{p} v}$. For daily effect, the value of $v=\omega$, but for seasonal effect, the value of $v=n$.

The value of $d a / d t$ above shows that the interruption of Yarkovsky effect depends on several parameters such as obliquity $(\gamma)$ and rotation, magnitude $(R)$, thermal's surface conductivity $(K)$, and distance from the Sun.

\section{DATA PROCESSING}

The numerical integration of n-body system is very sensitive to initial conditions, therefore it is impossible to analyze the orbital evolution of 1566 Icarus by use of one single numerical integration, especially from only one single set input of initial conditions. Small changes in one orbital element, will have an impact on changes in value for all other orbital elements as well, and this will change their traces in the evolution. The numerical integration process, has been done many times by generating many assumed different initial conditions of orbital element of asteroid 1566 Icarus. This process are defined as cloning process, and we make 100 clones of asteroid 1566 Icarus by generating random number from 0 to 1 according to each orbital element with $\sigma$ shown in Table 3. By using these values, we have 100 clones of asteroid 1566 Icarus that follow Gaussian distribution. All orbital elements are cloned by using probability density function $(p d f)$. After that, we compare the orbital dynamic of a 100 clones of asteroid 1566 Icarus using classical Newtonian with the orbital dynamics of asteroid 1566 Icarus within Yarkovsky effect from the nominal value of orbital element of asteroid 1566 Icarus from Table 1.

\section{Cloning Process}

Random number is a technique to randomly choose number within certain range. Cloning process has produced 100 clones of orbital element of asteroid 1566 Icarus with range value (orbital element $\pm \sigma$ ). All clones of orbital element were generated by adding and substracting orbital element value with $\sigma$ (standard deviation). If the value of orbital element is assumed $X$, then the value of $X$ is: $X-\sigma<X<X+\sigma$

Table 3 is the value of orbital element of asteroid 1566 Icarus with the value of standard deviation $(\sigma)$ for each orbital element. The data in table 3 was taken from database [8] at epoch 2456800.5 (23 May 2014).

Table 3. Orbital Element 1566 Icarus at Epoch 2456800.5 (23 May 2014) [8]

\begin{tabular}{cl}
\hline $\begin{array}{c}\text { Orbit element of } 1566 \\
\text { Icarus }\end{array}$ & \multicolumn{1}{c}{ Standard deviation } \\
\hline$a[\mathrm{au}]=1.07802$ & $\sigma_{a}[\mathrm{au}]=3.2116 \times 10^{-10}$ \\
$e=0.82677$ & $\sigma_{e}=6.6816 \times 10^{-8}$ \\
$i\left[^{\circ}\right]=22.83010$ & $\sigma_{i}\left[^{\circ}\right]=1.6181 \times 10^{-5}$ \\
$\omega\left[^{\circ}\right]=31.3542$ & $\sigma_{\omega}\left[^{\circ}\right]=8.3591 \times 10^{-6}$ \\
$\Omega\left[^{\circ}\right]=88.0275$ & $\sigma_{\Omega}\left[^{\circ}\right]=4.2726 \times 10^{-6}$ \\
\hline
\end{tabular}

All multiplied data by random number should follow probability density function $(p d f)$. The function is as follows:

$p d f=\mathbf{A} e^{-\left(\frac{x-\mu}{2 \sigma}\right)^{2}}$

with $\mu$ is the average of data value, $x$ is the original data value, $\sigma$ is a standard deviation data value and $\mathbf{A}$ is normalization within value $=1$. The $p d f$ value later be plotted on a curve of $p d f$ towards $\sigma$. The curve formed bell curve which shows valid to Gaussian distribution curve. Fig. 6 shows the curve $p d f$ on each orbital element of asteroid 1566 Icarus.

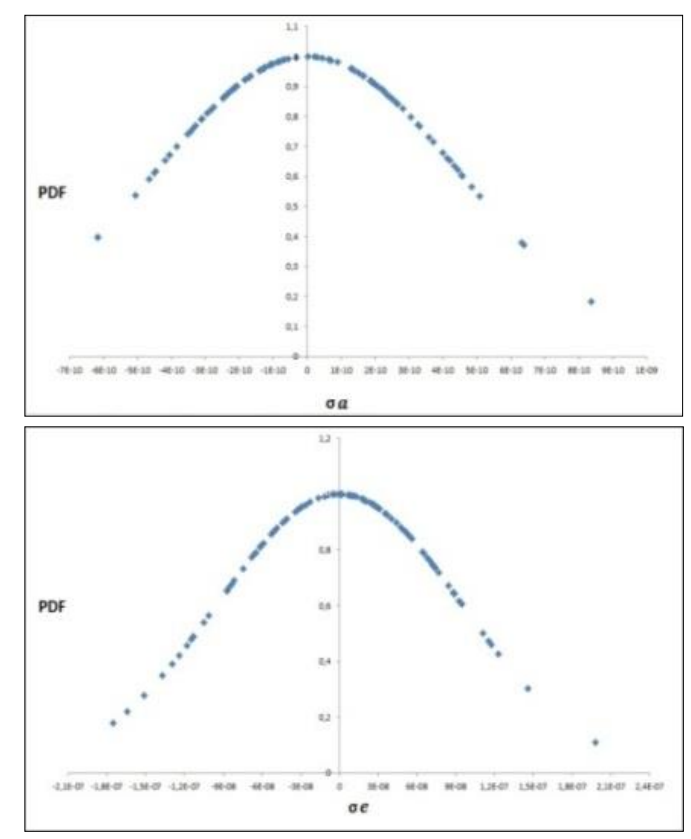




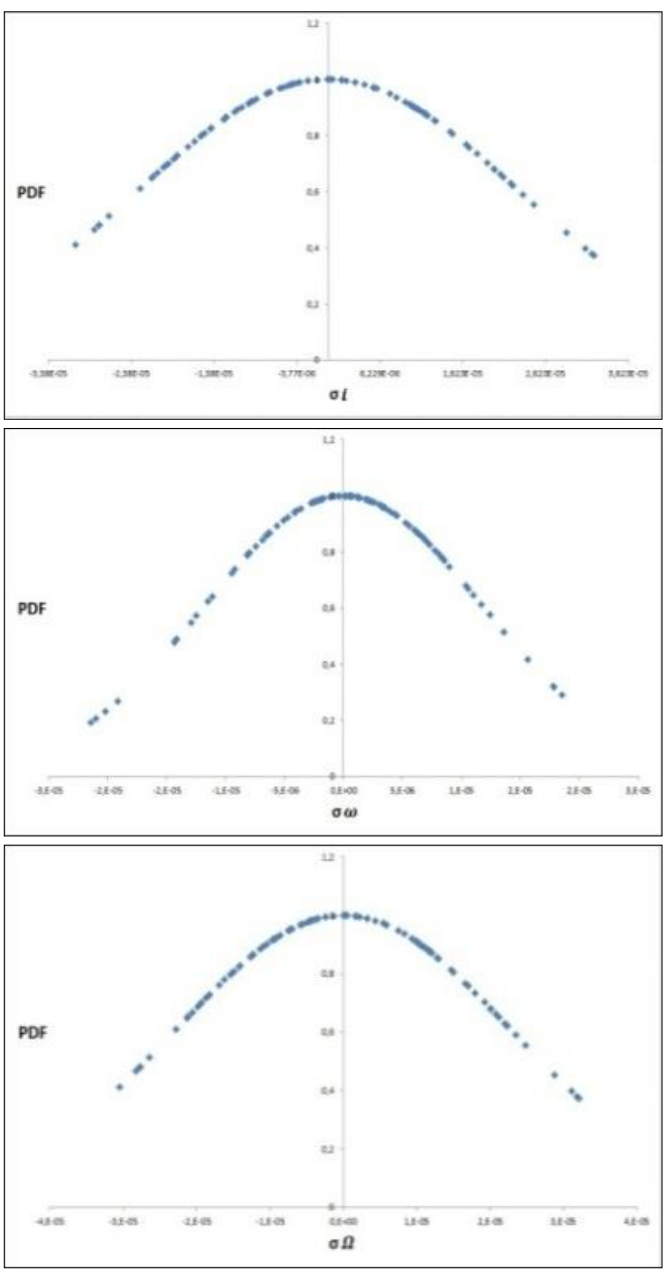

Figure 6. The curve of $p d f$ vs $\sigma$ of orbital element of asteroid 1566 Icarus.

All of a 100 value from each orbital element are combined to be 100 set clone data which later on called clone-1 up to clone-100. Clone-1 is set as orbital element data obtained from database [8]. Other clones are set of data from the combination of orbital element $\pm \sigma$.

\section{Integration Process}

The orbital dynamics of 1566 Icarus asteroid is investigated by numerical integration of the Classical Newtonian equations which only accommodates gravitation force and Yarkovsky effect equations. The Yarkovsky effect equations are equation of motions which includes Yarkovsky effect.

The integration process in this research took time frame at $t=0.0$ which is exactly at epoch 23 May 2014, up to $t=365.25 \times 10^{5}$ days or $10^{5}$ years ahead of the epoch. The time-step used in this integration is 0.879 days or 0.01 orbital period of planet Mercury. This integration process took two parts due to difference of integration method used, which are RMVS4 and RMVS4_ye. The result of both integration processes cover orbit element $a, e, i$, $\omega, \Omega$, and $M$ planets, and 100 clones testing particles of Asteroid 1566 Icarus.

For Classical Newtonian integration, the input data are 8 planets and 100 clones of asteroid 1566 Icarus. Testing particle consist of 100 clones set data Asteroid 1566 Icarus, which each of them covers $x$, $y, z, v_{x}, v_{y}$, dan $v_{z}$. On this integration, testing particles, Asteroid 1566 Icarus is considered has no mass. Parameter data covers range of integration time, $t$, and time step $d t$.

RMVS4_ye method enabling additional Yarkovsky effect in the integration process. Therefore, some parameters additional is required as input. The main input covers the same input with Classical Newtonian integration process which are planets, testing particles, and parameter data. Table 4 is the physical parameters data of Asteroid 1566 Icarus for Yarkovsky effect integration.

Table 4. Physical parameter of asteroid 1566 Icarus.

\begin{tabular}{lll}
\hline Physical parameter & Value & Source \\
\hline Radii & $1.3 \mathrm{~km}$ & {$[8]$} \\
Density of bulk & $\sim 3500 \mathrm{~kg} / \mathrm{m}^{3}$ & {$[4]$} \\
Surface density & $\sim 1500 \mathrm{~kg} / \mathrm{m}^{3}$ & {$[4]$} \\
Thermal conductivity & $\sim 0.168 \mathrm{~W} / \mathrm{m} / \mathrm{K}$ & {$[6]$} \\
Thermal capacity & $\sim 680 \mathrm{~J} / \mathrm{kg} / \mathrm{K}$ & {$[4]$} \\
Bond-Albedo & 0.33 & {$[8]$} \\
Emisivity & 0.92 & {$[4]$} \\
Rotation Periode & $2.273 \mathrm{jam}$ & {$[8]$} \\
Azimuth & 240,1 & {$[11]$} \\
Latitude & $+5^{\circ}$ & {$[11]$} \\
\hline
\end{tabular}

The result of the numerical integration from both processes then combined and plotted by SWIFTVIS software. This software was invented by Hal Levison and Martin Duncan particularly to process data result from SWIFT integrator. The plot shows the orbital dynamics of Asteroid 1566 Icarus according to both Classical Newtonian and involving Yarkovsky effect.

\section{RESULT}

Fig.7(a) to (e) shows the orbital dynamic of Asteroid 1566 Icarus. The black stripes show orbital dynamic from Classical Newtonian and the red stripes show orbital dynamic which includes Yarkovsky effect. 


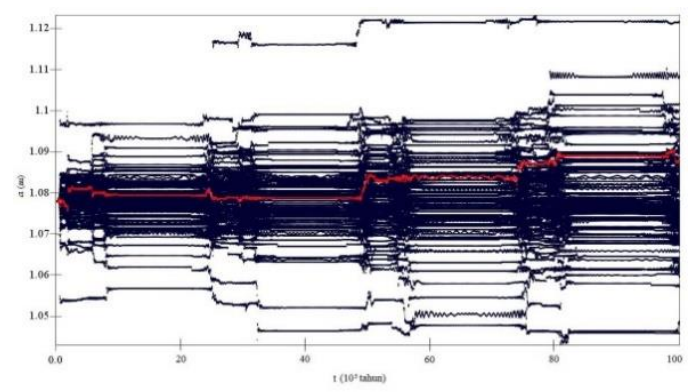

(a)

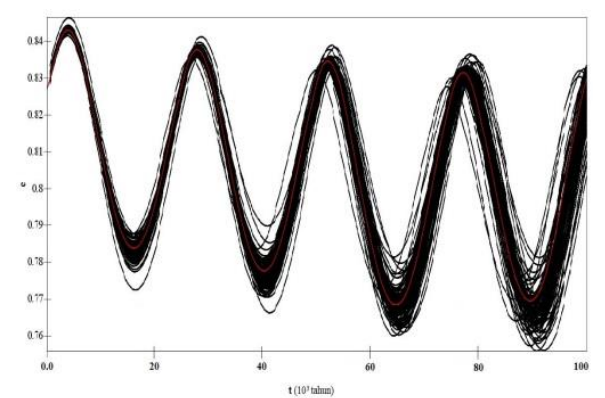

(c)

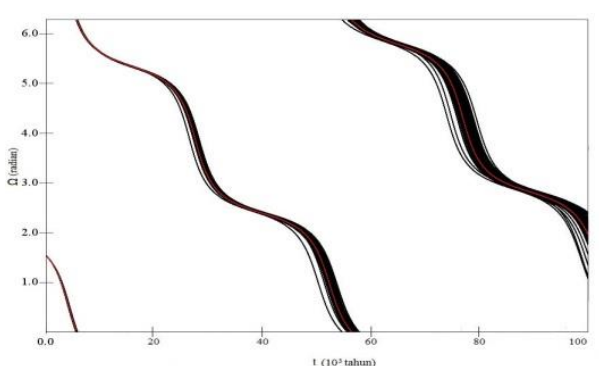

(e)

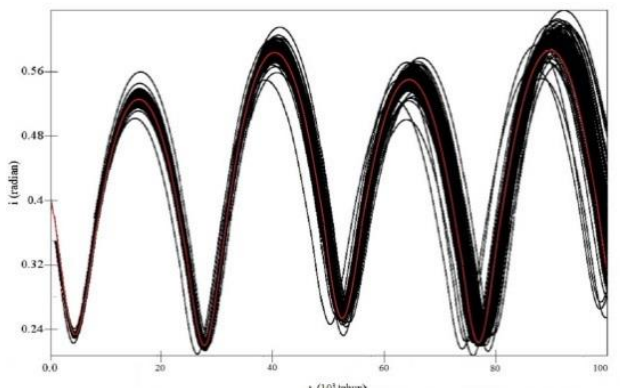

(b)

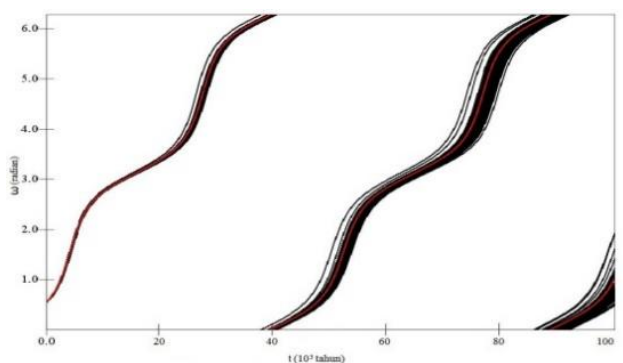

(d)

Figure 7. The dynamical of orbital-element of asteroid 1566 Icarus with Yarkovsky effect, respectively: (a) $a$ vs $t$, (b) $i$ vs $t$, (c) $e$ vs $t$, (d) $\omega$ vs $t$, (e) $\Omega$ vs $t$

From Fig. 7(a) to (e), the orbital dynamic of Asteroid 1566 Icarus lies within classical newtonian case range and was represented by 100 clones. Fig. 7(a) also shows the leap value in $a$, so Fig.7(b-e) consecutive in $i, e, \omega$, and $\Omega$. This is due to closeencounter of asteroid 1566 Icarus with major planets. An object is considered to have close- encounter with a planet when their distance is less than or equal to three times Hill-radii from the planets [5] [13]. The Hill-radii $R_{H}$ is represented by:

$R_{H}=a \sqrt[3]{\frac{m}{3 M}}$

With $R_{H}$ is Hill-radii, $m$ is mass of planet, and $M$ is mass of the Sun. The small object within the distance from the planet less than or equal to $3 R_{H}$, totally under gravitation of the planet, not the Sun.

\section{CONCLUSION}

With 100 clones of the orbital element of 1566 Icarus, using Yarkovsky effect or not, we find this asteroid will be stable for 100,000 years from 0 time (epoch 23 Mei 2014), with a small variation in the semimajor-axis $(a)$ and eccentricity (e), and will not escape from the Solar System or go inside to the Sun.

The asteroid 1566 Icarus suffers many close encounters with the inner-planets, it makes the 'jumps' of its orbital elements which can be seen in the dynamics of semimajor-axis. The variation of the semimajor-axis is not seen to be periodic, it changes in many ways. Those changes are caused by close encounters with 4 planets: Mercury, Venus, Earth and Mars, while its orbital plane stays at a high inclination $\left(i \approx 10^{\circ}-31^{\circ}\right)$. The close-encounters will change the dynamics of orbital elements. Actually not only $a$ is jumped, but all of the orbital elements 
are jumped too, but with a small scale make the jumped can not be seen,

Globally, there are no differences of the numerical integration results between Classical Newtonian method and by adding Yarkovsky effect, but the trends show us that there are differences on the amount and the timing of close-encounter events.

\section{ACKNOWLEDGMENT}

We are grateful to Institut Teknologi Bandung for give us the grant to get the opportunity to attend in Seminar Kontribusi Fisika, 16-17 December 2015 in Bandung, Indonesia.

\section{REFERENCES}

[1] G. Beekman; I.O. Yarkovsky and the Discovery of 'His' Effect, JHA, 37, Part 1, No. 126, 71-86, 2006

[2] R. P. Binzel, D. F. Lupishko, M. Di Martino, R. J. Whiteley, and G. J. Hahn, Physical Properties of Near-Earth Objects, in Bottke, W. F., Cellino, A., Paolicchi, P., and Binzel., R.P (eds.). Asteoids III, 2002

[3] W. F. Bottke, D. Vokrouhlicky, D. P. Rubincam, and M. Broz, The Effect of Yarkovsky Thermal Forces on The Dynamical Evolution of Asteroids and Meteoroids, in W. F., Cellino, A., Paolicchi, P., and Binzel., R.P (eds.). Asteroids III, 2002

[4] M. Broz, Yarkovsky Effect and the Dynamics of Solar System, PhD Thesis, Charles University,
Faculty of Mathematics and Physics Astronomical Institute, Prague, 2006.

[5] M. Cuntz and K. E. Yeage, On the Validity of the "Hill Radius Criterion" for the Ejection of Planets from Stellar Habitable Zones, Ap.J, 697, L86-L90, 2009

[6] M. Delbo et al., Thermal inertia of near-Earth asteroids and implications for the magnitude of the Yarkovsky effect, Icarus, 190, 236-249, 2007.

[7] http:/neo.jpl.nasa.gov/neo/groups.html

[8] http://ssd.jpl.nasa.gov/horizons.cgi

[9] https://upload.wikimedia.org/wikipedia/commo ns/thumb/5/51/YarkovskyEffect.svg/300pxYarkovskyEffect.svg.png)

[10] http://www.boulder.swri.edu/ hal/swift.html

[11] A. Kryszczynska et al., New Findings on Asteroid Spin-vector Distribution, Icarus, 192, 223-237, 2007

[12] C.R. Nugent et al., The Yarkovsky Drift's Influence on NEAs: Trends and Predictions with Neowise Measurements, A.J, 144, 75, U.S.A, 2012

[13] E. J. Opik; Celestial Mechanics: (Book Reviews: Interplanetary Encounters, Closerange Gravitational Interactions), Science, 194, issue 4265, 609-610, 1976

[14] E. Soegiartini, Perilaku Orbit Asteroid 1566 Icarus, $\mathrm{PhD}$ Thesis, Institut Teknologi Bandung, Bandung, 2012. 